\title{
Fiber Positioning and MRS Response
}

\author{
D. Beznosko, P. Polozov, V. Rykalin, G. Sellberg
}

\begin{abstract}
This paper provides experimental results on the dependence of the output signal from small-area silicon photodetectors, such as MRS (Metal-Resistive layerSemiconductor) photodiodes, on the fiber alignment.
\end{abstract}

\section{INTRODUCTION}

$\mathrm{T}$ HE new silicon photodetectors, such as MRS [1], offer a variety of advances in optical cell readout. Because of their miniature sizes $(1 \mathrm{~mm} \quad \mathrm{x} \quad 1 \mathrm{~mm})$ and presumable insensitivity to high magnetic fields, these sensors can be placed inside a detector, or even mounted on the scintillator, eliminating the need for the long fibers and optical connectors, thus reducing the light attenuation in them.

However, because the active area of the above detectors is, in many cases, comparable with the area of the signal-carrying fiber, an alignment issue becomes important. In the case when large number of the scintillator unit cells with MRS will be produced, the accuracy of the alignment poses constraint on the precision of the assembly of those cells and the automation of the process.

In this paper we present experimental data on the dependence of the output from MRS on the fiber alignment with the sensor. Scans with fiber moving along, away and angled to the sensor were conducted.

\section{EXPERIMENTAL SECTION}

\section{A. Apparatus Description}

A block diagram of the experimental setup used is shown in Fig. 1. Light signals from the green LED (peak emission at $\sim 510 \mathrm{~nm}$ ) via a clear fiber were supplied to the MRS and readout was measured using a Tektronix [2] TDS2024 oscilloscope. Position and movements of the fiber with respect to the sensor were accomplished using Newport [3] 462 Series XYZ-M Integrated Linear Stage (Fig. 2). This Stage allows

Manuscript received May 9, 2004.

P. Polozov is with ITEF, Moscow, Russia (e-mail: polozov@fnal.gov).

G. Sellberg is with Fermi National Accelerator Laboratories, Batavia, IL 60510 USA (telephone: 630-840-8630, email: sal@fnal.gov).

D. Beznosko, V. Rykalin are with Northern Illinois University, DeKalb, IL 60115 USA (telephone: 815-753-3504, email: rykalin@fnal.gov). achieving the linearity of travel better than $100 \mu \mathrm{rad}$ about any axis and reproducible return to the same point within accuracy of \pm 2.5 micron.

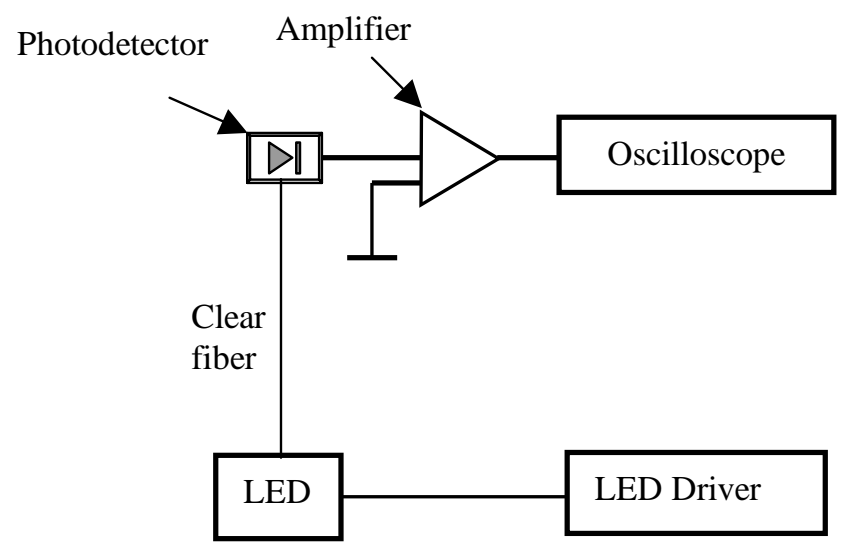

Fig. 1. Block diagram showing the apparatus used.

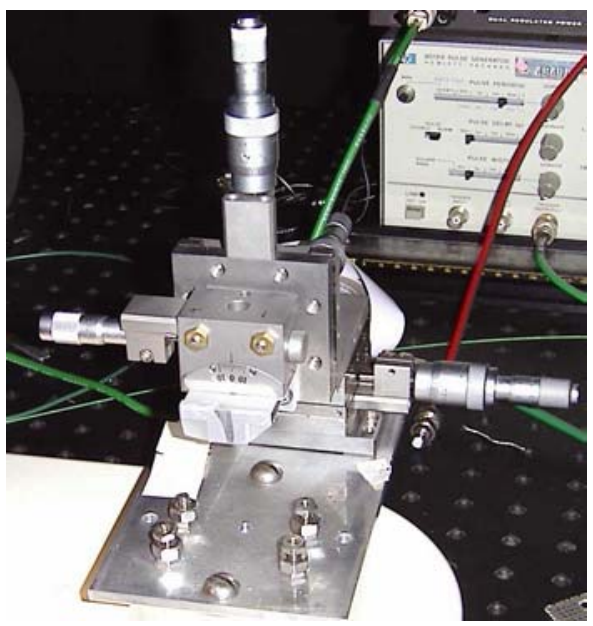

Fig. 2. 462 series XYZ-M used.

\section{B. Scans}

The following measurements were conducted using MRS sensor. Sensor responses with fiber moving along and away from it were obtained. For all of the following scans, unless stated otherwise, a $0.5 \mathrm{~mm}$ outer diameter clear fiber was used and the sensor itself was biased at $52 \mathrm{~V}$, chosen following the manufacturer's recommendations. 
Fig. 3a shows the result of the scan along the sensor in the direction indicated in Fig. 3b. A plateau corresponds to the region where the entire area of the fiber is within the photosensitive area of the sensor. Somewhat long tails on the far right and left sides are due to the light reflection off the protective shielding and the mount of the sensor, thus very small, but non-zero, value of the response is observed when the fiber moves completely away from the photosensitive area of the MRS. Another contributing effect is due to the fact that fiber is not pressed firmly onto the sensor area so that is could be moved along it without causing any damage. Hence, as the light signal exits the fiber, it forms a cone with somewhat larger cross-area at the surface of the sensor then the fiber itself would present. Precision of these measurements is approximately $\pm 12 \mathrm{mV}$ at each point. Positioning accuracy is \pm 2.5 micron. These values are the same for all further plots.

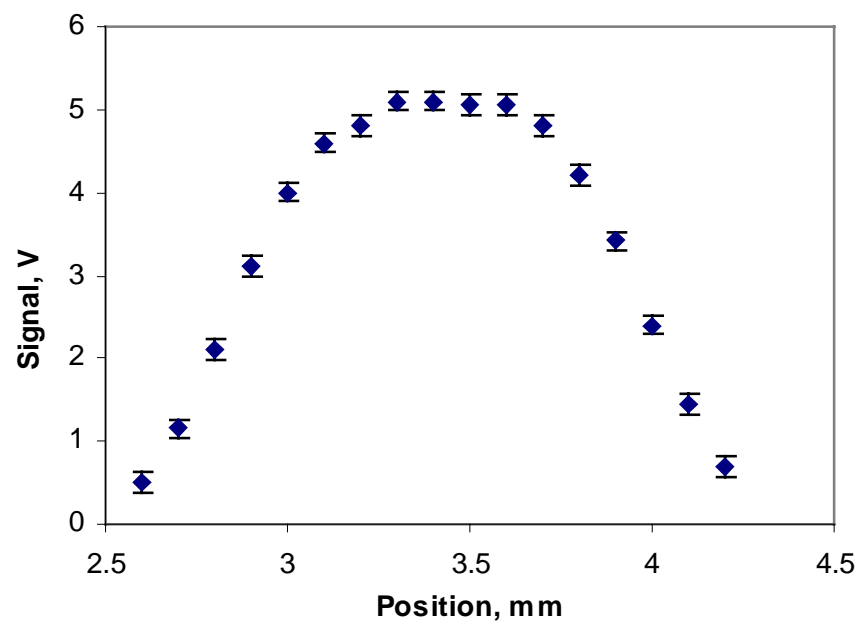

Fig. 3a. Output signal amplitude versus position of the fiber along the MRS sensor. Error bars are not shown so that the shape of the curve is visible.

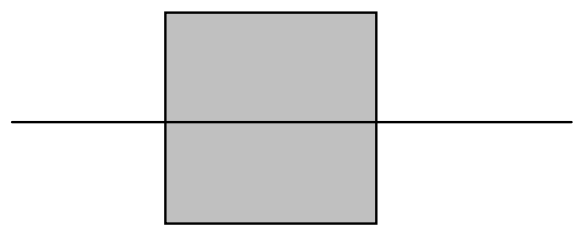

Fig. 3b. Sensor orientation and the direction of the scan (indicated by horizontal line).

In addition, measurements of the output signal amplitude versus the distance of the fiber away from the sensor were performed. Fig. 4 shows the results for this scan. Point at $0 \mathrm{~mm}$ corresponds to the fiber being physically in contact with MRS surface. Scan was performed with fiber positioned in the approximate center of the photosensitive area of the sensor, well within the plateau region (Fig. 3a).

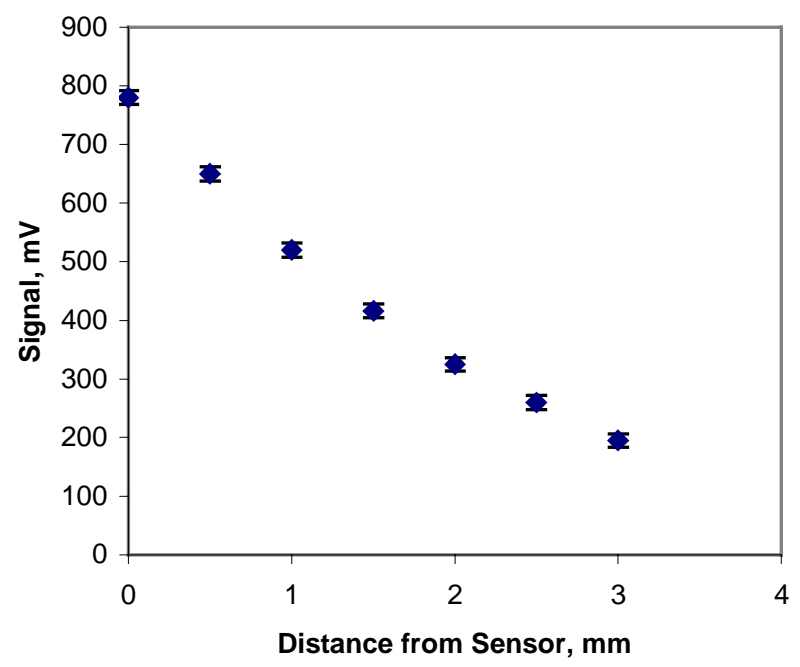

Fig. 4. Output signal amplitude versus fiber distance from the sensor.

Additional scan along the MRS sensor was performed using the $0.94 \mathrm{~mm}$ outer diameter clear fiber in order to see what is the dependence of the output signal amplitude on the position of the fiber being the size that is likely to be used for scintillating cell readout and which area is close to the area of the sensor itself. Fig. 5 shows the result of the measurement with $0.94 \mathrm{~mm}$ fiber. Note that there is a peak rather then a plateau in Fig. 5. It corresponds to fiber being completely centered on the sensor and is dominated by the contribution from the core of the fiber.

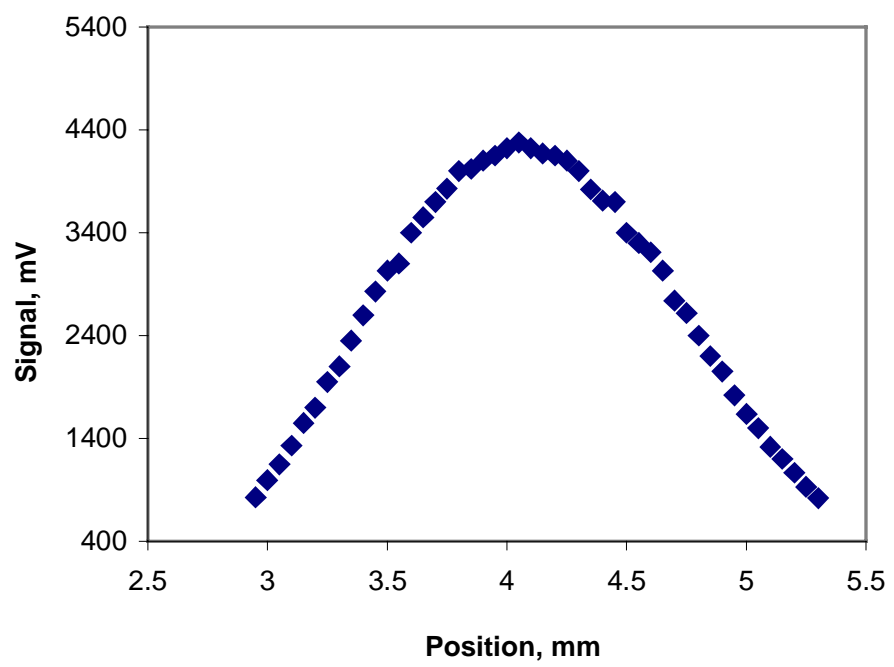

Fig. 5. Output signal amplitude versus position of the $0.94 \mathrm{~mm}$ fiber along the MRS sensor.

The dependence of the output signal on the fiber angle to the sensor was also measured. Fig. 6a shows the $0.5 \mathrm{~mm}$ fiber position during these measurements (done at the approximate center of the sensor), and Fig. 6b shows the result of that scan. 


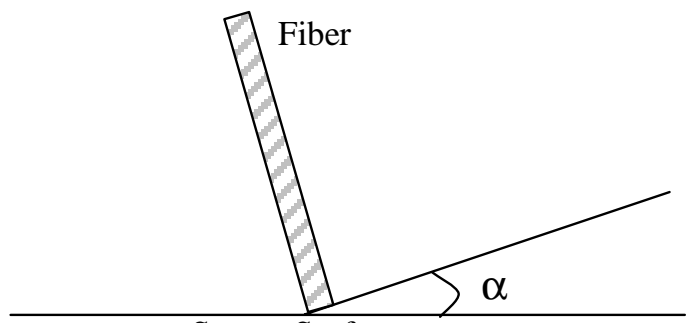

Sensor Surface

Fig. 6a. Fiber position with respect to the surface of the MRS.

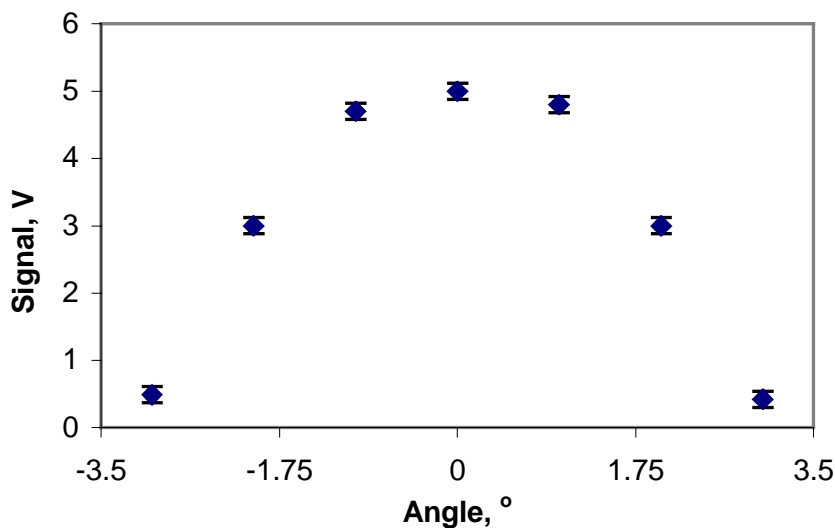

Fig. 6b. Output signal amplitude versus angle of the $0.5 \mathrm{~mm}$ fiber to the MRS sensor surface.

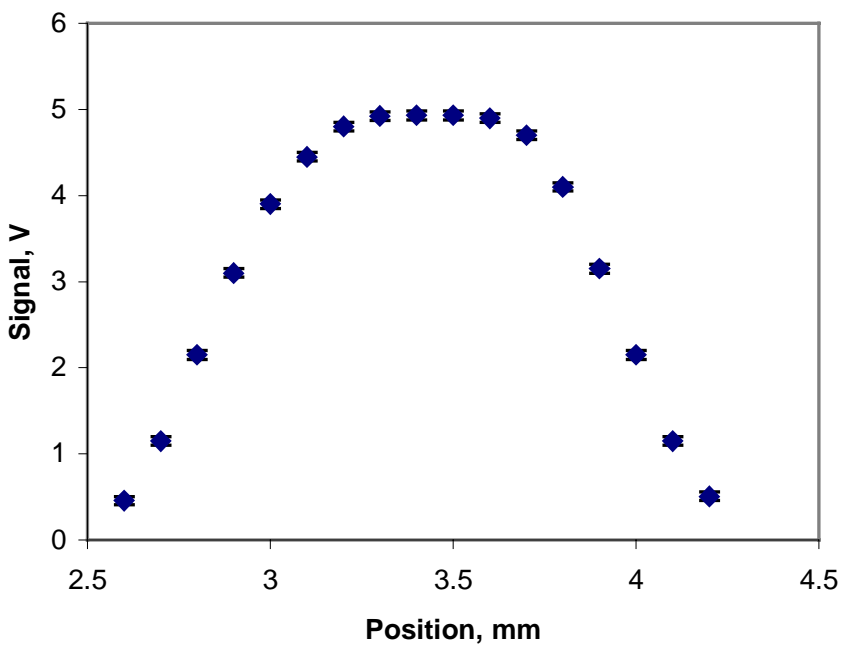

Fig. 7. Output signal amplitude versus position of the tilted at $\alpha=-1^{\circ} 0.5 \mathrm{~mm}$ fiber along the MRS sensor.

Finally, a scan along the sensor with the fiber tilted at $\alpha=-1^{\circ}$ was performed. Negative angle indicates the fiber tilt is to the opposite side of that in Fig. 6a. Results of that scan are shown in Fig. 7. Direction of the scan is the same as in Fig. 3b. The curve shows slight asymmetry that is due to the tilt. Fig. 8 is the scan at $\alpha=-2^{\circ}$ to illustrate that the width of the plateau is weakly dependent of the tilt angle.

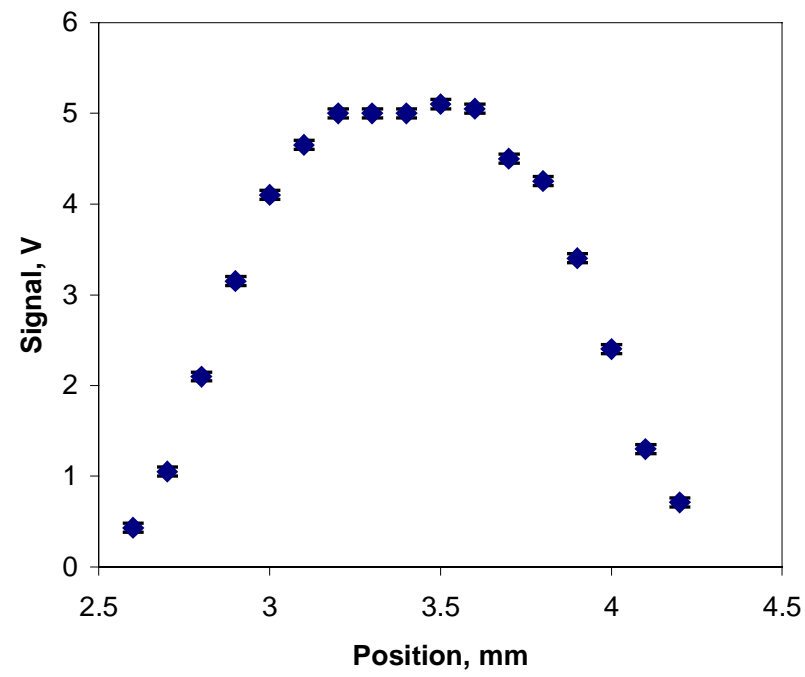

Fig 8. Output signal amplitude versus position of the tilted at $\alpha=-2^{\circ} 0.5 \mathrm{~mm}$ fiber along the MRS sensor.

\section{CONCLUSION}

Measurements indicate that out of all possible fiber misalignments with the sensor, the tilt of the fiber is the least damaging in terms of the level of the output signal from the sensor. The tilt of the fiber from the normal to the sensor surface of 1 degree results in only $4 \%$ loss of the output. On the other hand, an air gap of $0.5 \mathrm{~mm}$ between fiber and the sensor decreases the output by $16.7 \%$.

For the fibers with areas smaller then that of a sensor there is a plateau of full signal detection so there is some freedom in the alignment along the sensor, as long as the detector covers the entire area of the fiber. For thicker fibers with areas that are comparable with that of the sensor, placement of the fiber in the center of the sensor becomes an important issue. The performed manual scan results indicate that process of the alignment and of the final coupling between fiber and Silicon sensor is possible and may be automated using the potential and experience of the MicroDetector Group at Silicon Detector Facility (at FNAL).

\section{ACKNOWLEDGMENT}

We thank Anna Pla-Dalmau for providing support for the measurements. 


\section{REFERENCES}

[1] V. Golovin et al., Limited Geiger-Mode Silicone Photodiode With Very High Gain. Nucl. Phys. Proc. Suppl. 61B: 347-352, 1998

[2] Tektronix Inc, 14200 SW Karl Braun Drive, P.O. Box 500, Beaverton, OR, 97077 USA.

[3] Newport Corporation, 1791 Deere Avenue, Irvine, CA, 92606 USA. 